\title{
Efectos del Attention Process Training (APT) en la percepción de mejora atencional en personas diagnosticadas de esquizofrenia
}

RESUMEN: El objetivo de este trabajo es analizar el efecto de un programa diseñado para mejorar problemas atencionales, en la percepción subjetiva de mejora atencional de veinticuatro pacientes esquizofrénicos.

PALABRAS CLAVE: Rehabilitación; Procesos atencionales; Percepción subjetiva; Esquizofrenia
ABSTRACT: The goal of this study was to investigate the effect of an attention training program designed to improve attention problems on subjective perception of attentional improvement in twentyfour schizophrenic patients.

KEY WORDS: Rehabilitation; Attention Process; Subjective perception; Schizophrenia

\section{Introducción.-}

Se ha demostrado de forma consistente que los déficit cognitivos son una característica central en la esquizofrenia y uno de los principales factores que contribuyen al deterioro funcional y social de estos pacientes $(1,2)$. Por ello, algunos autores, al observar que estas alteraciones parecen tener más impacto que los síntomas positivos y negativos en las actividades de la vida diaria de los pacientes con esquizofrenia, han puesto de manifiesto la necesidad de considerar las alteraciones cognitivas como un objetivo clínico prioritario (3).

La evidencia con respecto a que los esquizofrénicos pueden aprender nuevas habilidades en entornos de entrenamiento altamente estructurados (1), ha servido de base para la aplicación de programas de rehabilitación cognitiva en estos pacientes. Los pocos trabajos que versan sobre este tipo de rehabilitación se centran, en su mayoría, en los procesos atencionales. Una de las razones por las que suele ser esta función el objeto de las intervenciones es porque algunos déficit atencionales son considerados marcadores de vulnerabilidad del trastorno, además de ser una de las principales quejas en su vida diaria (4).

Algunos estudios pioneros demostraron que entrenando directamente los procesos atencionales en pacientes esquizofrénicos se podía mejorar su rendimiento atencional. Por ejemplo, Benedict y Harris (5) analizaron el efecto de la práctica repetida con tareas cognitivas presentadas por ordenador, encontrando que las personas que recibieron entrenamiento mejoraron sus tiempos de reacción, mientras que esto no ocurrió en las personas que no fueron entrenadas. 
En el trabajo de Benedict y Harris se observó una mejoría en pruebas de evaluación distintas a las utilizadas durante la fase de entrenamiento. No obstante, esta generalización de los resultados no siempre se obtiene. De hecho, en la mayoría de los estudios analizados, aunque el rendimiento en las propias tareas utilizadas en la rehabilitación mejora, esta mejoría no se generaliza a las pruebas de la evaluación. Por ejemplo, Triano-Antidormi (6) no encontró mejoría en las tareas empleadas en la evaluación en siete pacientes que fueron entrenados con el Attention Process Training, comparados con un grupo control formado por otros siete pacientes que no recibieron entrenamiento; no obstante, el rendimiento de los pacientes en las tareas utilizadas en la intervención fue mejorando a lo largo del entrenamiento. Este mismo efecto aparece en el estudio de Benedict y cols (7) y en el de Medalia y cols (8). Parecería, por tanto, que la atención es una función dependiente de la tarea, en el sentido de que ésta mejora en aquellas tareas que se ejecutan de forma repetida pero esa mejoría no se generaliza a otras tareas distintas a las practicadas.

El planteamiento general de este tipo de estudios de rehabilitación cognitiva es el siguiente: primero, se evalúa al paciente con diversas pruebas neuropsicológicas y de procesamiento de la información. Después, se realiza el entrenamiento y, al finalizarlo, se evalúa de nuevo en esas u otras pruebas semejantes. De esta forma, el rendimiento obtenido en las pruebas antes del entrenamiento se compararía con el obtenido después del mismo. Además, algunos estudios comparan estos datos con los alcanzados por otros pacientes que no han sido entrenados. El objetivo de todo esto es averiguar el efecto que específicamente el entrenamiento, y no variables extrañas (como por ejemplo, el paso del tiempo o la medicación), puede tener en el funcionamiento cognitivo.

Un aspecto que permanece sin analizar en los estudios efectuados hasta ahora es el efecto que el entrenamiento tiene en la percepción del paciente de mejora de la atención. Es decir, independientemente del efecto que el entrenamiento pueda tener en las pruebas neuropsicológicas utilizadas para evaluar la atención, es importante saber cómo cree el paciente que se está viendo afectada su cognición como consecuencia del entrenamiento. Podría ocurrir, por ejemplo, que una persona mejore su puntuación en una prueba de ejecución continua y no tenga sensación de mejoría en su capacidad para prestar atención en una tarea continua en el tiempo. $\mathrm{O}$ al contrario, que tras recibir entrenamiento para mejorar su capacidad atencional manifieste que le es más fácil seguir una conversación cuando hay jaleo a su alrededor, pero que su rendimiento en una prueba que evalúe atención selectiva no mejore. Estas posibles discrepancias pueden ser importantes a la hora de valorar la eficacia diferencial de las intervenciones y el costo-beneficio de las mismas. Por ejemplo, en un estudio sobre rendimiento en pruebas de memoria, Cerviño y Vázquez (9) demostraron que las valoraciones sobre el rendimiento cognitivo de los pacientes eran diferentes para los propios pacientes, los familiares y los psicólogos responsables de estos pacientes.

El objetivo de este estudio es averiguar si personas diagnosticadas de esquizofrenia entrenadas en atención percibirán una mayor mejoría en sus procesos atencionales, además de mejorar su rendimiento en pruebas que evalúan atención, comparados con pacientes no entrenados. 
El entrenamiento seleccionado para realizar este estudio fue el Attention Process Training (APT). Además de ser un programa que ha mostrado su eficacia en personas con daño cerebral $(10,11)$, está diseñado y articulado en torno a un modelo sobre el funcionamiento de la atención.

Para analizar un posible efecto de generalización del rendimiento entre las diferentes tareas, es importante tener en cuenta el tipo de pruebas que se utilizan en la evaluación. Debe incluirse material que evalúe el mayor rango posible de niveles atencionales en sus diversas modalidades sensoriales. Por ello, se escogieron pruebas que evaluaran los diferentes niveles atencionales que entrena el APT. De esta forma, se seleccionaron pruebas que evalúan atención sostenida, selectiva, alternante y dividida; con material visual y auditivo. Además, se diseñó una prueba específica con el objetivo de conocer la percepción del paciente sobre el efecto del entrenamiento en sus procesos atencionales.

Nuestras hipótesis de trabajo son que si el APT es un entrenamiento efectivo entonces mejorará el rendimiento en las pruebas de evaluación y también aumentará la percepción subjetiva de mejora atencional del paciente.

\section{Método}

\section{SUJETOS}

En el estudio participaron veintinueve pacientes diagnosticados de esquizofrenia según el DSM-IV (12), procedentes de los Centros de Rehabilitación Psicosocial "Martínez Campos", "Los Cármenes" y "Retiro" de la Comunidad Autónoma de Madrid y de la asociación AMAFE. Del total de sujetos que participaron en el estudio sólo se analizaron los datos de veinticuatro, el resto fue excluido del análisis por diversas razones, tales como hospitalización por descompensación psicótica, abandono voluntario del estudio o diagnóstico confuso.

Para poder ser incluidos en el estudio los pacientes debían cumplir no sólo los criterios diagnósticos DSM-IV sino presentar quejas de problemas atencionales y no participar en ningún programa de rehabilitación cognitiva durante la realización del estudio ni previo al mismo. Fueron excluidos aquellos pacientes que presentaban conductas agresivas, sospecha de abuso o dependencia de alcohol u otras drogas, o evidencia de enfermedad neurológica. En el momento del estudio todos los pacientes recibían tratamiento farmacológico con neurolépticos.

Los sujetos fueron asignados aleatóriamente a uno de los dos grupos experimentales: uno recibió entrenamiento con el APT y el otro no recibió entrenamiento (no recibió ningún otro tratamiento adicional ni placebo). Finalmente, trece pacientes formaron parte del grupo entrenado y once del grupo control. 


\section{PRUEBAS DE EVALUACIÓN}

Sintomatología y nivel de funcionamiento global:

La sintomatología se valoró con la puntuación total de la Escala Breve de Evaluación Psiquiátrica (BPRS-Brief Psychiatric Rating Scale)(13). Durante el transcurso de una entrevista se valoran los síntomas presentes en los últimos tres días basándose en la información aportada por el sujeto y la conducta observada. Contiene veinticuatro ítems, cada uno de los cuales se puntúa en una escala de siete puntos de gravedad $(1=$ no presente $7=$ extremadamente grave $)$.

La información obtenida en la entrevista fue también usada para completar la Escala para la Evaluación de Síntomas Positivos (SAPS- Scale for the Assessment of Positive Symptoms)(14) y la Escala para la Evaluación de Síntomas Negativos (SANSScale for the Assessment of Negative Symptoms)(15). La SAPS está diseñada para evaluar los síntomas positivos de la esquizofrenia. Está formada por treinta y cuatro ítems agrupados en las siguientes categorías: Alucinaciones, Delirios, Conducta extravagante y Desorden formal del pensamiento. La segunda escala tiene como objetivo evaluar los síntomas negativos de la esquizofrenia. Consta de veinticinco ítems agrupados en cinco áreas: Afecto embotado, Alogia, Abulia-apatía, Anhedonia-insociabilidad y Atención. En ambas escalas se obtiene una puntuación global para cada categoría, que tiene en cuenta la naturaleza y la gravedad de los diversos tipos de síntomas que engloban el apartado. Para su valoración se utiliza una escala de seis puntos de gravedad.

El nivel de funcionamiento global se estimó con la Escala de Evaluación de la Actividad Global (EEAG) incluida en el DSM-IV (12). Esta escala puntúa el funcionamiento global del sujeto teniendo en cuenta su actividad psicológica, social y laboral, en un continuo que va de la salud plena [1] a la incapacidad total [100].

Funcionamiento cognitivo

Además de evaluarse el estado mental, sintomatología y funcionamiento global, se seleccionaron pruebas para cubrir diferentes niveles atencionales así como el funcionamiento cognitivo general.

Estado cognitivo global

Se utilizó el Mini-Examen del Estado Mental (Mini-Mental State Examination) (16). Esta prueba consta de cinco apartados: orientación témporo-espacial, memoria inmediata, capacidad atencional y de cálculo, memoria demorada y lenguaje. La puntuación total se obtiene sumando la puntuación obtenida en cada uno de los ítems. La puntuación máxima de la prueba es 30 puntos. Puntuaciones menores de 24 indican un deterioro cognitivo leve, y puntuaciones menores de 15 sugieren la presencia de demencia severa.

Atención sostenida

Test de Ejecución Continua (CPT- Continuous Performance Test). Se emplearon dos versiones del CPT, suministrados en forma de paquete informático para ordenador 
(17). En ambas modalidades aparece, en el centro de un monitor de ordenador, un dígito por segundo. El objetivo de la tarea consiste en presionar el botón de un mando para juegos cada vez que aparezca en la pantalla el número cero. En primer lugar, se aplicó un CPT, tipo CPT-X, empleando como estímulos dígitos perfectamente enfocados. El segundo CPT, aplicado tras un breve descanso, era un CPT de estímulos degradados (igual que el anterior pero el estímulo se presenta más borroso al estar aleatorizado, para cada ensayo, el 40\% de los píxeles del fondo de la pantalla). En el análisis de datos se utilizaron la razón de aciertos, la razón de falsas alarmas (apretar el botón a un número que no es el cero) así como los tiempos medios de reacción de los aciertos y de las falsas alarmas del total de ensayos experimentales.

Parte $A$ del Test de Trazado (TMT- Trail Making Test)(18). Consta de una hoja con veinticinco números, rodeados cada uno por un círculo. El objetivo de la tarea es unir los números, lo más rápido posible, de forma secuencial y en orden ascendente. Se analiza el tiempo empleado y el número de errores cometido.

Tarea de cancelación (19). Esta versión modificada del test de papel y lápiz Toulouse-Pieron (20) consiste en una hoja llena de cuadrados con una pequeña raya que los atraviesan, o tocan, en alguno de sus lados. El objetivo consiste en tachar, durante un período de tiempo determinado, los cuadrados que tengan una raya que lo atraviesen en la parte superior. Se analizaron el número total de aciertos, omisiones y de falsas alarmas.

Atención selectiva

Fue evaluada con una prueba de escucha dicótica (21). A través de unos auriculares, se presentan de forma simultánea palabras diferentes por el oído izquierdo y por el oído derecho. El objetivo de la tarea consiste en repetir, en voz alta, todas las palabras que aparecen por el oído derecho. Aparece un total de 120 pares de palabras. Se analizaron el número total de aciertos y de errores (palabras que no aparecieron y palabras que se presentaron por el oído izquierdo).

Atención alternante

La Parte B del Test de Trazado (TMT- Trail Making Test) (18). Consiste en una hoja en la que hay letras (de la A a la L) y números (del 1 al 13) rodeados por un círculo. El objetivo es unir, de forma alternante, números y letras (1-A-2-B-3-C...). Se analiza el tiempo empleado y el número de errores cometido.

\section{Atención dividida}

Para evaluar el funcionamiento en atención dividida se aplicaron las pruebas de cancelación y escucha dicótica de forma simultánea. Los valores empleados en el análisis de datos corresponden a los mismos utilizados en su aplicación por separado.

Control mental

Tarea de Adición Serial Auditiva Pautada (PASAT- Paced Auditory Serial Addition Task) (22). Es un test auditivo en el que se presentan secuencialmente dígitos 
que se deben ir sumando conforme al siguiente formato: el primero con el segundo, el segundo con el tercero, el tercero con el cuarto, y así sucesivamente. Consta de cuatro partes. Cada una tiene la misma secuencia de dígitos pero varían en la velocidad de presentación de los números. Se analizaron el número de aciertos y falsos positivos de la primera y cuarta parte.

Funcionamiento atencional en la vida cotidiana

Se evaluó a través del Cuestionario de Atención de la Vida Cotidiana (EAQEveryday Attention Questionnaire) (23). Es una prueba heteroaplicada diseñada para detectar déficit atencionales en la vida diaria. Consta de cinco apartados: Atención sostenida, Atención selectiva para tareas fáciles, Atención selectiva para tareas difíciles, Atención dividida y Atención en situaciones comunes (por ejemplo, leer mientras escucha la radio). En el análisis de datos se utilizó la puntuación media de los ítems de cada apartado.

Percepción subjetiva de mejoría atencional

Cuestionario sobre Percepción de Mejora Atencional (19). Es una prueba diseñada específicamente por nosotros y está formada por doce ítems en los que se pregunta al paciente si se ha producido mejoría, o empeoramiento, en diferentes aspectos de la atención, tales como hacer varias cosas a la vez o concentrarse cuando hay ruido alrededor. Cada ítem se puntúa en una escala tipo Likert de siete puntos $(0=$ Ha empeorado mucho; 3 = igual; $6=$ Ha mejorado mucho).

Se diseñaron dos modelos del cuestionario: uno para el grupo entrenado y otro para el grupo control. Ambos constan de los mismos ítems, pero mientras que las personas que recibieron entrenamiento tienen que valorar su funcionamiento actual comparado con su funcionamiento antes del entrenamiento, las del grupo control deben valorar su funcionamiento en el transcurso del tiempo especificado (dependiendo del tiempo que haya transcurrido entre su primera y segunda evaluación).

Ejercicios de entrenamiento:

Para realizar el entrenamiento en atención se seleccionó el Attention Process Training (APT) (24), el cual fue desarrollado para mejorar déficit atencionales en personas con daño cerebral (10).

El APT es un programa de intervención individual que trata cuatro niveles de atención (sostenida, selectiva, alternante y dividida) a través de diferentes tareas organizadas jerárquicamente en orden creciente de dificultad (tareas de cancelación visual y auditiva, de control mental y de vida cotidiana).

En la tabla 1 se presenta de forma resumida las tareas administradas. 


\section{ATENCIÓN SOSTENIDA}

Tachar un objetivo en tareas de cancelación de figuras y números (prueba de papel y lápiz).

Cintas de atención 2-8 (series de números presentados auditivamente debiéndose dar un golpe cuando aparece el objetivo).

Serie de números (restar mentalmente números).

Tareas de la vida diaria (buscar algo en guías, mapas, etc.).

\section{ATENCIÓN SELECTIVA}

Tachar un objetivo en tareas de cancelación de figuras y números (prueba de papel y lápiz con fondo distractor).

Cintas de atención 9-16 (series de números presentados auditivamente con un fondo de ruido, debiéndose dar un golpe cuando aparece el objetivo).

Tareas de la vida diaria (buscar algo en guías, mapas, etc., con música de fondo).

\section{ATENCIÓN ALTERNANTE}

Tachar un objetivo, que cambia cada quince segundos, en tareas de cancelación de figuras y números (prueba de papel y lápiz).

Alternar sumas y restas, tachar números pares e impares, y tareas tipo Stroop de tamaño y posición.

\section{ATENCIÓN DIVIDIDA}

Ejecutar simultáneamente la prueba de cancelación visual y la auditiva.

Emparejar Cartas (clasificar las cartas conforme al palo y además poner al revés la que contenga determinada letra).

Tareas de la vida diaria (jugar a un juego).

Tabla 1: Tareas de intervención empleadas en el entrenamiento.

Procedimiento

Tras obtener su consentimiento para participar en el estudio, el paciente era entrevistado por uno de los investigadores (BL) para confirmar el diagnóstico de esquizofrenia de su historial clínico. En la entrevista, se evaluaba su estado mental para descartar la posible existencia de una enfermedad neurológica, y se aplicaba el cuestionario de Atención de la Vida Cotidiana para confirmar la existencia de quejas atencionales.

Todos los pacientes fueron evaluados por el mismo clínico durante cuatro sesiones de una hora cada una, durante un período de dos semanas.

Tras la evaluación, los sujetos eran asignados de forma aleatoria a uno de los dos grupos. Los sujetos pertenecientes al grupo entrenado recibían entrenamiento con el APT. Las tareas de entrenamiento fueron administradas por tres estudiantes de último curso de Psicología de la Universidad Complutense de Madrid, quienes habían sido entrenados 
específicamente para el estudio. Cada paciente tenía asignado el mismo terapeuta para llevar a cabo todas las sesiones de su entrenamiento.

La primera sesión se invertía en explicar cómo iba a ser el proceso. Además, el terapeuta debía de localizar los ejercicios del programa con los que el paciente debía iniciar el entrenamiento. Esto debía hacerse con cada tipo de material (cancelación visual, cancelación auditiva y control mental). El punto de inicio era aquel ejercicio en el que el paciente fallaba dos veces consecutivas.

Cada una de las sesiones posteriores tenían un patrón uniforme: durante los primeros minutos de cada sesión se explicaba en qué consiste la atención y cómo pueden detectarse problemas en ella. Seguidamente, se practicaba repetidamente con cada tipo de material.

Los ejercicios eran presentados en grado de dificultad creciente, de tal forma que los pacientes comenzaban con tareas a niveles relativamente fáciles e iban avanzando, conforme mejoraba su actuación, a niveles más difíciles. Se daba un ejercicio como válido cuando lo realizaba bien tres veces consecutivas. Durante el entrenamiento se hacía constantemente una revisión sistemática del rendimiento y se daba retroalimentación al paciente.

El tratamiento duró una media de 48.5 sesiones por paciente $\left(S_{x}=10.4\right)$. Dependiendo de la capacidad y disponibilidad del paciente, se realizaba de dos a tres sesiones semanales. El tiempo medio invertido por sesión fue de 42.6 minutos $\left(\mathrm{S}_{\mathrm{x}}=8.7\right)$.

Una vez finalizada la intervención, los pacientes eran evaluados de nuevo con las mismas pruebas que las utilizadas antes del entrenamiento. En la segunda evaluación se añadió el Cuestionario sobre Percepción de Mejora Atencional. En ella se preguntaba al paciente si percibía mejoría o empeoramiento en los diferentes ítem de los que consta el cuestionario.

Los sujetos del grupo control fueron evaluados con las mismas pruebas que el grupo entrenado y en el mismo orden. Para determinar cuándo un paciente de este grupo debía ser evaluado por segunda vez, cada uno de los pacientes del grupo control fue emparejado con uno del grupo entrenado en las variables de sexo, edad y duración de la enfermedad. De esta forma, si un paciente tardaba siete meses en completar su entrenamiento, su pareja del grupo control sería evaluada por segunda vez seis meses después de la primera evaluación.

Los pacientes del grupo control no recibieron entrenamiento en atención pero participaron en los programas del centro, al igual que hicieron los participantes del grupo entrenado.

\section{Análisis de datos}

El análisis estadístico de los datos se realizó mediante el programa SPSS-PC, versión 10.0. El procedimiento de cada análisis se describirá en el capítulo de resultados. 
ORIGINALES Y REVISIONES

Resultados

En la tabla 2 se muestran las características demográficas, clínicas y de funcionamiento de los dos grupos de sujetos participantes en el estudio. No se han encontrado diferencias significativas entre los grupos experimentales en las variables de sexo, edad, duración de la enfermedad, número de hospitalizaciones previas, dosis de neurolépticos recibida (equivalencia en clorpromacina), estado mental y nivel de funcionamiento global.

En relación con la sintomatología, tampoco se encontraron diferencias significativas entre los grupos excepto en el apartado de conducta extravagante del SAPS $[F(1)=5.507$; $\mathrm{p}<0.05]$, en la que el grupo entrenado (Grupo APT) obtuvo una puntuación más elevada que la del grupo control.

\begin{tabular}{|l|c|c|}
\hline & $\begin{array}{c}\text { Grupo APT } \\
(\mathrm{n}=13)\end{array}$ & $\begin{array}{c}\text { Grupo control } \\
(\mathrm{n}=11)\end{array}$ \\
\hline Sexo, \% varones & 92 & 73 \\
Edad, media de años $\left(\mathrm{S}_{\mathrm{x}}\right)$ & $34.7(8.4)$ & $32.2(8.9)$ \\
Duración de la enfermedad, media de años $\left(\mathrm{S}_{\mathrm{x}}\right)$ & $13.9(5.4)$ & $12.4(10)$ \\
$\mathrm{N}^{0}$ de hospitalizaciones previas, media $\left(\mathrm{S}_{\mathrm{x}}\right)$ & $4.3(4.5)$ & $3.4(3.9)$ \\
Dosis de neurolépticos, media clorpromacina $\left(\mathrm{S}_{\mathrm{x}}\right)$ & $490.3(219.4)$ & $387.4(254)$ \\
Mini-Mental, puntuación total media $\left(\mathrm{S}_{\mathrm{x}}\right)$ & $27.4(2)$ & $26.4(1.9)$ \\
BPRS, puntuación total media $\left(\mathrm{S}_{\mathrm{x}}\right)$ & $37.3(7.9)$ & $37.4(13.6)$ \\
$\mathrm{SAPS}-$ puntuación media alucinaciones $\left(\mathrm{S}_{\mathrm{x}}\right)$ & $0,8(1.6)$ & $1.6(2.3)$ \\
$\quad$ puntuación media delirios $\left(\mathrm{S}_{\mathrm{x}}\right)$ & $2.8(1.7)$ & $2.8(2.3)$ \\
$\quad$ puntuación media conducta extravag. $\left(\mathrm{S}_{\mathrm{x}}\right) *$ & $0.9(1.2)$ & $0(0)$ \\
$\quad$ puntuación media desorden formal $\left(\mathrm{S}_{\mathrm{x}}\right)$ & $1.6(1.4)$ & $1.1(1.6)$ \\
SANS - puntuación media afecto embotado $\left(\mathrm{S}_{\mathrm{x}}\right)$ & $2.5(1.7)$ & $2.4(2)$ \\
$\quad$ puntuación media alogia $\left(\mathrm{S}_{\mathrm{x}}\right)$ & $1.6(1.5)$ & $1.7(1.8)$ \\
$\quad$ puntuación media abulia-apatía $\left(\mathrm{S}_{\mathrm{x}}\right)$ & $1.8(1.4)$ & $2(1.7)$ \\
$\quad$ puntuación media anhedonia $\left(\mathrm{S}_{\mathrm{x}}\right)$ & $3.4(0.9)$ & $3(1.2)$ \\
puntuación media atención $\left(\mathrm{S}_{\mathrm{x}}\right)$ & $1(1.3)$ & $0,6(1.2)$ \\
EEAG & $45.5(15.9)$ & $46.8(20.8)$ \\
\hline
\end{tabular}

$* \mathrm{p}<0.05$

Tabla 2: Características demográficas y clínicas de los pacientes. 
ORIGINALES Y REVISIONES

En la tabla 3 se presentan, para cada grupo, las puntuaciones medias obtenidas en las pruebas cognitivas en las dos evaluaciones.

\begin{tabular}{|c|c|c|c|c|c|}
\hline \multirow[t]{2}{*}{ Prueba } & \multirow[t]{2}{*}{ Valor } & \multicolumn{2}{|c|}{ Grupo entrenado } & \multicolumn{2}{|c|}{ Grupo control } \\
\hline & & $\begin{array}{l}\text { Puntuación } \\
\text { previa } \\
\text { media }\left(S_{x}\right)\end{array}$ & $\begin{array}{l}\text { Puntuación } \\
\text { posterior } \\
\text { media }\left(S_{x}\right)\end{array}$ & $\begin{array}{l}\text { Puntuación } \\
\text { previa } \\
\text { media }\left(\mathrm{S}_{\mathrm{x}}\right)\end{array}$ & $\begin{array}{l}\text { Puntuación } \\
\text { posterior } \\
\text { media }\left(\mathrm{S}_{\mathrm{x}}\right)\end{array}$ \\
\hline \multirow[t]{4}{*}{ CPT simple } & Razón aciertos & $1(0)$ & $0.9(0.1)$ & $0.9(0.2)$ & $0.8(0.1)$ \\
\hline & TR aciertos mseg. & $610.5(87.7)$ & $610.8(92.2)$ & $633.8(74.1)$ & $643.9(126.7)$ \\
\hline & Razón falsas alarmas & $0(0)$ & $0(0)$ & $0(0.1)$ & $0(0)$ \\
\hline & TR falsas alarmas & $540.4(273.7)$ & $521.5(180.4)$ & $526.2(103.8)$ & $478.4(156.1)$ \\
\hline \multirow[t]{4}{*}{ CPT degradado } & Razón aciertos & $0.6(0.3)$ & $0.6(0.3)$ & $0.5(0.3)$ & $0.5(0.3)$ \\
\hline & TR aciertos mseg. & $739(240.2)$ & $691(154.7)$ & $754.5(169.7)$ & $727.6(170.5)$ \\
\hline & Razón falsas alarmas & $0.1(0.3)$ & $0.1(0.1)$ & $0.1(0.1)$ & $0.1(0.1)$ \\
\hline & TR falsas alarmas & $606.4(120.1)$ & $580.7(126.5)$ & $645.8(72.9)$ & $608.4(128.4)$ \\
\hline Tarea de cancelación & Total correctas & $95.4(6.4)$ & $97.8(4.1)$ & $91.2(5.9)$ & $92.4(8.3)$ \\
\hline (atención dividida) & Total falsas alarmas & $0.7(1.8)$ & $0(0.1)$ & $0.8(1.4)$ & $0.4(1.2)$ \\
\hline Escucha dicótica & Total correctas & $101.5(21.6)$ & $106.6(15.6)$ & $94(22.5)$ & $88.4(25.2)$ \\
\hline (atención dividida) & Total errores & $3.5(3.39$ & $2.5(3.5)$ & $4.1(3)$ & $2.9(2.5)$ \\
\hline \multirow[t]{4}{*}{ PASAT } & Total correctas $1 .^{\mathrm{a}}$ & $30(9.6)$ & $33.4(12.5)$ & $23.9(8.8)$ & $33.3(7.5)$ \\
\hline & Falsas alarmas $1^{\text {a }}$ & 14.7 (12.1) & $6.2(2.5)$ & $6.3(3)$ & $5.7(5.7)$ \\
\hline & Total correctas $4^{\mathrm{a}}$ & $14(8)$ & $21.1(4.3)$ & $15.4(7.9)$ & $14.8(6.6)$ \\
\hline & Falsas alarmas $4^{\mathrm{a}}$ & $18.1(17.9)$ & $4.5(2.5)$ & $39.7(8.9)$ & $10.8(16.2)$ \\
\hline \multirow[t]{4}{*}{ Test de Trazado } & Tiempo total, seg. (a) & $59.6(29.8)$ & $60.8(40.6)$ & $72.4(45.9)$ & $69.5(46.4)$ \\
\hline & Total errores (a) & $0.1(0.3)$ & $0.1(0.3)$ & $0.2(0.4)$ & $0.4(0.9)$ \\
\hline & Tiempo total seg. (b) & $150.4(120.7)$ & $166.2(164.5)$ & $229.2(185)$ & $189(118.5)$ \\
\hline & Total errores (b) & $0.9(1.1)$ & $0.9(1.4)$ & $2.1(1.4)$ & $1.4(1.2)$ \\
\hline Cuestionario de & Media apartado 1 & $1.8(0.8)$ & $1.8(0.7)$ & $1.8(0.8)$ & $1.3(0.9)$ \\
\hline Atención de Vida & Media apartado 2 & $2.3(0.9)$ & $2.3(0.7)$ & $2.6(0.5)$ & $2.8(0.5)$ \\
\hline \multirow[t]{3}{*}{ Cotidiana } & Media apartado 3 & $3.2(0.9)$ & $3(0.8)$ & $3.1(0.6)$ & $3.6(0.9)$ \\
\hline & Media apartado 4 & $2.2(0.5)$ & $2.3(0.8)$ & $2.4(0.8)$ & $3.1(0.9)$ \\
\hline & Media apartado 5 & $2.3(0.9)$ & $1.9(1)$ & $2.7(0.7)$ & $2.7(0.5)$ \\
\hline
\end{tabular}

*p $<0.05$
Tabla 3. Puntuaciones medias calculadas para cada grupo en las pruebas cognitivas aplicadas antes y después del tratamiento. 
Con el objetivo de analizar el efecto del entrenamiento en las pruebas atencionales empleadas en la evaluación, se hizo una ANOVA 2x2 de medidas repetidas (Grupo entrenado versus Grupo control x Primera evaluación versus Segunda evaluación) con cada una de las variables especificadas en la descripción de las pruebas.

Los resultados obtenidos tras el análisis muestran que no existen diferencias significativas en ninguna de las variables analizadas, con la excepción del número total de respuestas correctas en la tarea de escucha dicótica cuando ésta se realiza junto con la prueba de cancelación $[\mathrm{F}(1)=4.434 ; \mathrm{p}<0.05]$. Un análisis detallado de las medias en esta variable (ver tabla 3 ) nos permite concluir que la puntuación media del grupo entrenado se mantiene igual mientras que la del grupo control mejora.

El mismo tipo de análisis fue realizado para la medicación, el nivel de funcionamiento global y los síntomas, encontrándose que no había interacción significativa ni efectos principales.

Para analizar nuestra segunda hipótesis de trabajo, esto es, que los pacientes entrenados tendrán una mayor percepción de mejora de sus procesos atencionales que los pacientes no entrenados, se hizo un análisis de varianza con cada uno de los doce ítems del Cuestionario de Percepción de Mejora Atencional entre el grupo entrenado y el grupo control (en la tabla 4 se muestran los valores medios en los ítems para cada grupo).

\begin{tabular}{|l|c|c|}
\hline ÍTEMS DEL CUESTIONARIO SOBRE & $\begin{array}{c}\text { Grupo APT } \\
\text { Pedia }\left(\mathrm{S}_{\mathrm{x}}\right)\end{array}$ & $\begin{array}{c}\text { Grupo control } \\
\text { Media }\left(\mathrm{S}_{\mathrm{x}}\right)\end{array}$ \\
\hline 1. Hacer varias cosas a la vez & $4.2(1)$ & $3.6(1.4)$ \\
2. Concentrarse cuando hay jaleo alrededor & $4.3(1.1)$ & $3.8(1.6)$ \\
3. Enterarse de lo que le dicen & $4.3(1.1)$ & $3.9(1.7)$ \\
4. Enterarse de lo que lee & $3.9(1)$ & $3.7(1.8)$ \\
5. Seguir una conversación & $3.8(1.12)$ & $3.9(2)$ \\
6. Seguir el hilo del argumento de una película & $3.6(1.4)$ & $3.5(1.7)$ \\
7. Cometer menos errores & $3.7(1.1)$ & $4.2(1.2)$ \\
8. Encontrar más fácilmente cosas que busca* & $4.5(1.1)$ & $3.2(1.2)$ \\
9. No ser tan despistado & $4.1(1.4)$ & $3.6(1.7)$ \\
10. Recordar citas & $4.7(1.1)$ & $3.6(1.7)$ \\
11. Acordarse de devolver algo prestado & $4.9(1.1)$ & $4(1.5)$ \\
12. Acordarse de dar un recado & $4.4(1.2)$ & $3.5(1.7)$ \\
\hline
\end{tabular}

$* \mathrm{p}<0.05$

Tabla 4: Puntuaciones medias calculadas para cada uno de los ítems del Cuestionario de Percepción de Mejora Atencional. 
Los resultados obtenidos señalan que solamente existen diferencias significativas entre ambos grupos en el ítem $8[\mathrm{~F}(1)=5.72 ; \mathrm{p}<0.05]$, el cual hace referencia a la facilidad para encontrar cosas que busca. Tal y como se puede apreciar en las medias que aparecen recogidas en la tabla 4, el grupo que recibió entrenamiento con el APT es el que percibe mayor mejoría en este ítem.

En ambos grupos, las puntuaciones medias en todos los ítems se sitúan en torno a 4, valor que significa percibir haber mejorado un poco.

\section{Discusión}

Nuestra primera hipótesis de trabajo era que el APT sería efectivo en mejorar la atención en personas diagnosticadas de esquizofrenia. Los datos obtenidos indican que el entrenamiento no mejoró el funcionamiento atencional de los pacientes que recibieron entrenamiento respecto a los pacientes control. Con respecto a esta primera conclusión, es importante hacer una matización. Por el tipo de estudio diseñado lo correcto es afirmar que los pacientes entrenados con el APT no mejoraron su rendimiento en las pruebas atencionales empleadas en la evaluación.

La ausencia de resultados no puede explicarse por diferencias en la dosis de tratamiento recibido puesto que todos los pacientes que recibieron entrenamiento finalizaron el mismo. Al ser un tratamiento individualizado, unos acabaron antes y otros necesitaron más tiempo para completar el programa pero lo importante es que todos llegaron hasta el final del mismo. Cada uno de los pacientes empezó el entrenamiento en su propio nivel atencional, generalmente un nivel de dificultad sencillo, y poco a poco, a través de la práctica repetida, fueron avanzado a niveles más complejos. Así pues, aunque los pacientes fueron mejorando su rendimiento en las tareas empleadas en el entrenamiento con el APT, esta mejora no se extendió a medidas diferentes a las del propio entrenamiento. Observamos que si bien hay una mejoría de la atención en la muestra de pacientes entrenados en este estudio, ésta se encuentra vinculada a la tarea. Este efecto de práctica en la ejecución de ciertas pruebas, tales como el Test de Aprendizaje Verbal de California y otras tareas de memoria y perceptivas, también ha sido encontrado en otros estudios $(25,26)$.

Nuestra primera conclusión es, por tanto, que el funcionamiento atencional de los pacientes mejoró a través de los ejercicios utilizados en el entrenamiento, pero esta mejoría no se generalizó a otras tareas al no obtenerse mejoría en las pruebas utilizadas en la evaluación, las cuales eran distintas a las empleadas en el entrenamiento.

Con respecto a las diferencias significativas encontradas en el número de aciertos de la escucha dicótica en la condición dividida, éstas no pueden ser atribuidas al APT, puesto que la puntuación media del grupo entrenado en esa variable tras la intervención fue la misma que antes del entrenamiento. Es el grupo control quien, paradójicamente, mejoró significativamente. Puesto que ambos grupos no se diferenciaron a lo largo del estudio en la medicación, la sintomatología ni el funcionamiento global, no podemos considerar a estas variables como responsables de la inesperada mejora encontrada en el grupo control. 
El otro objetivo del estudio era averiguar si los pacientes entrenados percibirían una mayor mejoría en sus procesos atencionales que los que no recibieron entrenamiento. Con respecto a este punto, debemos indicar que como el rendimiento en las pruebas atencionales empleadas en la evaluación de los pacientes entrenados no mejoró, no cabría esperar que percibieran mejoría en sus procesos atencionales.

$\mathrm{Si}$ analizamos detalladamente las medias obtenidas en cada uno de los ítems en ambos grupos, podemos observar que todas ellas se desvían positivamente del punto medio [M=3] el cual significa "no cambio". Las medias se sitúan en torno a un valor de 4, lo que significa que perciben haber mejorado un poco. Dado que esto se observa en ambos grupos no podemos concluir que sea debido al APT, sino que será debido a un elemento común en ambos grupos, tales como el terapeuta u otras actividades del centro, pero esta explicación es sólo hipotética pues hay medidas que puedan confirmarla.

No sabemos si esa leve mejoría percibida en la atención es exclusiva de la atención o si corresponde a una sensación general de mejora y, por tanto, aparece en otras variables. Para averiguar esto habría sido necesario diseñar un instrumento que midiera la percepción subjetiva de mejora en otros ámbitos (memoria, estado de ánimo, etc.).

Podría haber existido la posibilidad de que el entrenamiento hubiera tenido un efecto placebo. Esto implicaría que, a pesar de no observarse mejoría en las pruebas empleadas en la evaluación, el hecho de recibir entrenamiento para mejorar la atención podría haber sido suficiente para que los pacientes percibieran mejora en su capacidad atencional. Después de los datos derivados del estudio podemos concluir que el hecho de recibir un entrenamiento específico en atención no sirve, por sí solo, para que el paciente considere que sus procesos atencionales mejoran. Un problema añadido de este tipo de intervenciones neurocognitivas es que no hay una base teórica suficiente que guíe de un modo pautado y articulado su desarrollo y aplicación. Aunque hay algunos pasos prometedores en este sentido en ámbitos como, por ejemplo, la neurorehabilitación motora (27), creemos que la utilidad de estas intervenciones está aún limitada por la falta de conexión entre los hallazgos del laboratorio y la intervención aplicada.

\section{Agradecimientos}

Este estudio ha sido financiado por una beca del Ministerio de Educación y Ciencia concedida a la primera autora (subprograma de Ayudas para el Intercambio de Personal Investigador entre Industrias y Centros Públicos de Investigación) y por una ayuda de la Comunidad Autónoma de Madrid concedida al segundo autor (Ayuda de Investigación del Plan Regional de Investigación). Nos gustaría agradecer a los Centros de Rehabilitación Psicosocial de la Comunidad Autónoma de Madrid, AMAFE, y a los estudiantes que realizaron los entrenamientos, su constante apoyo y colaboración. 


\section{BIBLIOGRAFÍA}

(1) Bellack, A.S.; Gold, J.M.; Buchanan, R.W. "Cognitive rehabilitation for schizophrenia: problems, prospects, and strategies" Schizophrenia Bulletin, 1999, 25, 257-274.

(2) Velligan, D.I. y otros. "Preliminary evaluation of Cognitive Adaptation Training to compensate for cognitive deficits in schizophrenia". Psychiatric Services, 1996, 47, 415-417.

(3) Green, M.F. "What are the functional consequences of neurocognitive deficits in schizophrenia?" American Journal of Psychiatry, 1996, 153, 321-330.

(4) Vázquez, C.; López, B.; Florit, A. "Procesamiento de la información y esquizofrenia: hallazgos empíricos y bases teóricas para la rehabilitación”. En J.A. Aldaz y C. Vázquez (comps). Esquizofrenia: fundamentos psicológicos y psiquiátricos de la rehabilitación. Madrid, Siglo XXI, 1996, pp. 23-55

(5) Benedict, R.; Harris, A. "Remediation of attention deficits in chronic schzizophrenic patients: a preliminar study". British Journal of Clinical Psychology, 1989, 28, 187-188.

(6) Triano-Antidormi, L. A controlled study of the cognitive and clinical efficacy of attention training in schizophrenia. 1996. Tesis doctoral sin publicar. Universidad McMaster, Hamilton (Ontario)

(7) Benedict, R. y otros. "Effects of attention training on information processing in schizophrenia". Schizophrenia Bulletin, 1994, 20, 537-546.

(8) Medalia, A. y otros. "Effectiveness of Attention Training in schizophrenia". Schizophrenia Bulletin, 1998, 24, 147-152.

(9) Cerviño, M.J.; Vázquez, C. Everyday memory deficits in schizophrenic patients: A comparison of assessments made by patients, psychologists, and first-degree relatives. Cambridge (Octubre, 1995). I International Congress on Psychological Treatments in Schizophrenia.

(10) Sohlberg, M.M.; Mateer, C.A. "Effectiveness of an attention-training program". Journal of Clinical and Experimental Neuropsychology, 1987, 9, 117-130.

(11) Park, N.W.; Proulx, G; Wanda, M.T. "Evaluation of the Attention Process Training Programme". Neuropsychological Rehabilitation, 1999, 9, 135-154.

(12) American Psychiatric Association. DSM-IV. Manual diagnóstico y estadístico de los trastornos mentales. (4 edición). Washington: American Psychiatric Association. 1995.

(13) Overall, J.E.; Gorham, D.R. "The Brief Psychiatric Rating Scale". Psychological Reports, 1962, 10, 799-812.

(14) Andreasen, N. Scale for the Assessment of Positive Symptoms (SAPS). Iowa City: University of Iowa College of Medicine. 1984.

(15) Andreasen, N. Scale for the Assessment of Negative Symptoms (SANS). Iowa City: University of Iowa College of Medicine. 1983

(16) Folstein, M.F.; Folstein, S.E.; McHugh, P.R. "“Mini-Mental State': a practical method for grading the cognitive state of patients for the clinical". Journal of Psychiatric Research, $1975 ; 12 ; 189-198$.

(17) Nuechterlein, K.H.; Asarnow, R.F. UCLA Continuous Performance Test (CPT) Program for IBM-PC-compatible microcomputers, version 1. Unpublished computer program. University of California, Los Angeles. 1987.

(18) Reitan, R.M.; Davidson, L.A. Clinical neuropsychology: current status and applications. Nueva York: Hemisphere. 1974.

(19) López Luengo, B. Rehabilitación cognitiva en esquizofrenia: aplicación del APT (Attention Process Training). Tesis doctoral no publicada. Universidad Complutense de Madrid. 2000. 
(20) Toulouse, E.Y.; Pieron, H. Toulouse-Pieron: prueba perceptiva y de atención manual. Madrid: TEA. 1972.

(21) Vázquez, C. y otros (1990). "Attentional performance and positive vs negative symptoms in schizophrenia". En P.J. Drenth; J.A. Sergeant; J. Takens (Eds.), European Perspectives of Psychology (Vol. 3), Nueva York, John Wiley, pp. 91-106.

(22) Gronwall, D. "Paced auditory serial addition task: a measure of recovery from concussion". Perceptual and Motor Skills, 1977, 44, 367-373.

(23) Martin, M. "Ageing and patterns of change in everyday memory and cognition". Human Learning, 1986, 5, 63-74.

(24) Sohlberg, M.M.; Mateer, C.A. Attention Process Training (APT). Puyallup (WA): Association for Neuropsychological Research and Development. 1986.

(25) Hawkins, K.A.; Wexler, B.E. "California Verbal Learning Test practice effects in a schizophrenia sample”. Schizophrenia Research, 1999, 39, 73-78.

(26) Wexler, B.E. y otros. "Normal neurocognitive performance after extended practice in patients with schizophrenia". Schizophrenia Research, 1997, 26, 173-180.

(27) Taub, E.; Uswatte, G.; Elbert, T. (2002). "New treatments in neurorehabilitation founded on basic research". Nature Reviews Neuroscience, 3, 228-236.

\section{AUTORES}

*Profesora Ayudante del Departamento de Psicología de la Universidad de Jaén

**Profesor Titular de la Facultad de Psicología de la Universidad Complutense de Madrid (Departamento de Psicología Clínica)

\section{CORRESPONDENCIA}

Dra. Beatriz López Luengo

Departamento de Psicología - Universidad de Jaén

Campus Las Lagunillas s/n, 23071 - Jaén

Correo electrónico: blopez@ujaen.es

Tel.: 953002663

Fax: 953012197

Fecha recepción: 25-09-02 